\title{
Grau de Satisfação do Doente e do MGF com a Consulta de Teledermatologia do CHVNG/E
}

\author{
Diogo Teixeira', MD; Jorge Lopes?', MD; Ana Cristina Sousa ${ }^{7}$, MD; Miguel Costa ${ }^{7}, M D$; Eduarda O. Ferreira ${ }^{7}$, MD; Armando Baptista ${ }^{7}$, \\ MD; Paulo Varela', MD \\ 'Serviço de Dermatologia do Centro Hospitalar Vila Nova de Gaia/Espinho, Portugal
}

RESUMO - Introdução: A Teledermatologia (store and forward) tem sido usada para melhorar a triagem de pedidos no âmbito do Serviço Nacional de Saúde, e para dar resposta, numa minoria de casos, a situações simples. O pedido de consulta de Dermatologia via Consulta Tempo e Horas passou a ser obrigatoriamente acompanhado de foto(s) clínica(s), de acordo com o Despacho 6280/2018 de 28 de junho de 2018. Pretendemos avaliar a satisfação de doentes e médicos de família relativamente à consulta de teledermatologia em tempo diferido. Material e Métodos: Foi realizado um inquérito aos doentes sujeitos a consulta de teledermatologia durante o primeiro trimestre de 2018, com posterior análise retrospetiva do registo clínico. Foi também realizado um inquérito online aos médicos de Medicina Geral e Familiar pertencentes aos Agrupamentos de Centros de Saúde da área de referência do Centro Hospitalar Vila Nova de Gaia/Espinho. Resultados: Dos 153 doentes inquiridos, obteve-se resposta em $27,5 \%(n=42)$. Lesões pigmentadas corresponderam a 54,8\% $(n=23)$ do total das consultas. Foi proposto tratamento em $42,9 \%(n=18)$. Os doentes referiram preferir a consulta presencial em $90,2 \%(n=38)$. Obtiveram-se 82 respostas por parte dos médicos de família. Metade dos inquiridos concorda com a obrigatoriedade de anexação de fotografias clínicas, ainda que esta leva a um acréscimo de $11.21 \pm 8.84$ minutos ao tempo de referenciação. Quando é feita uma consulta de Teledermatologia, $47,6 \%(n=39)$ dos médicos referem que o problema do doente fica apenas parcialmente tratado. Conclusão: A utilização da Teledermatologia para consulta não presencial apenas resolve uma minoria das situações clínicas e não se apresenta como uma medida satisfatória para os doentes.

PALAVRAS-CHAVE - Consulta Remota; Dermatologia;Médicos de Família; Telemedicina.

\section{Patient and Family Doctor Satisfaction with Teledermatology Consultations in CHVNG/E}

ABSTRACT - Introduction: Teledermatology (store and forward) has been used to improve priority screening for hospital consultations and to respond, in a minority of cases, to simple situations. The Dermatology consultation request via Consulta Tempo e Horas must now be accompanied by clinical photo(s), according to Despacho 6280/2018 of 28 June 2018 . We intend to evaluate the satisfaction of patients and family doctors regarding teledermatology consultation. Material and Methods: A survey was carried out on patients subjected to teledermatology consultation during the 1st quarter of 2018, with a retrospective analysis of the clinical record. An online survey was also carried out on family doctors belonging to the Agrupamentos de Centros de SaúdeACeS of the Centro Hospitalar Vila Nova de Gaia/Espinho reference area. Results: Of the 153 patients surveyed, response was obtained in $27.5 \%(n=42)$. Pigmented lesions corresponded to $54.8 \%(n=23)$ of the total consultations. Treatment was proposed in $42.9 \%$ $(n=18)$. Patients would prefer in-person consultation in $90.2 \%(n=38)$ of cases. Eighty-two responses were obtained from family doctors. Half of the responders agree with the mandatory attachment of clinical photos to the consultation request, even if this leads to an increase of $11.21 \pm 8.84$ minutes to the request time. When a teledermatology consultation is made, $47.6 \%$ ( $n=39$ ) of family doctors report that the patient's problem is only partially treated. Conclusion: Teledermatology consultation (store and forward) only solves a minority of clinical situations. Furthermore, it is not considered a satisfactory solution for patients. KEYWORDS - Dermatology; Physicians, Family; Remote Consultation; Telemedicine.

Correspondência: Diogo Manuel Santos Teixeira Serviço de Dermatologia

Centro Hospitalar Vila Nova de Gaia/Espinho

R. Conceição Fernandes $\mathrm{S} / \mathrm{N}$

4434-502 Vila Nova de Gaia, Portugal

E-mail: diogomsantosteixeira@gmail.com

DOI: https://dx.doi.org/10.29021/spdv.78.3.1190
Recebido/Received 2020/04/17

Aceite/Accepted 2020/08/01

Publicado/Published 2020/09/30

(C) Autor (es) (ou seu (s) empregador (es)) 2020 Revista SPDV. Reutilização permitida de acordo com CC BY-NC. Nenhuma reutilização comercial.

(C) Author(s) (or their employer(s)) 2020 SPDV Journal. Re-use permitted under CC BY-NC. No commercial re-use. 


\section{Artigo Original}

\section{INTRODUÇÃO}

Telemedicina (TM) é definida como a utilização de tecnologias de comunicação aplicada ao ramo da medicina, para prestação de serviços médicos à distância. ${ }^{1}$ Com a crescente evolução da tecnologia, no que toca a rapidez, qualidade e quantidade de dados transmitidos, é expectável que a telemedicina venha a ter um papel cada vez mais preponderante na prática médica, especialmente para melhor acessibilidade aos cuidados médicos em regiões geograficamente remotas e com cuidados médicos deficitários.

A teledermatologia (TD) refere-se à telemedicina aplicada para cuidados dermatológicos. Começou a ser descrita na literatura durante a década de 90, com estudos na Noruega e nos EUA em populações rurais e de difícil acesso aos cuidados de saúde.

Dada a prevalência elevada de problemas dermatológicos, e a escassez de dermatologistas, ${ }^{2-6}$ a teledermatologia surge como uma eventual solução para esse problema.

A TD divide-se em 3 modalidades tecnológicas: Teleconsulta em tempo real, que consiste em videoconferência entre dois profissionais de saúde, na presença do doente (requer equipamento próprio, bem como a necessidade de conciliação de agendas de três pessoas); teleconsulta em tempo diferido (store and forward), mais utilizada, em que se procede ao armazenamento e envio de fotografias e informação clínica relevante para o dermatologista, com resposta posterior $^{1}$ e a modalidade híbrida.

A TD pode ser utilizada em 3 modelos assistenciais: telerrastreio - em que é feita uma triagem dos pedidos de referenciação hospitalar, classificando os doentes por prioridades de acordo com a gravidade das possíveis hipóteses diagnóstico, encaminhando-os também para a consulta de subespecialidade própria, se for caso disso; teleconsulta em que o dermatologista faz consultadoria; e monitorização.

A teledermatologia em tempo diferido apresenta-se uma modalidade mais atrativa. De facto, requer menos custos de equipamento (requer apenas uma câmara fotográfica) e maior flexibilidade, não necessitando a coordenação da agenda de três pessoas (dermatologista, médico de família e doente). No entanto, existem várias desvantagens desta modalidade, que resultam da falta de interação com o doente. 5 Nesse aspeto, a TD em tempo real pode apresentar algumas vantagens.

Atualmente, a TD tem sido cada vez mais utilizada com projetos em Espanha, ${ }^{7}$ França, ${ }^{5}$ Suíça, Holanda, ${ }^{8}$ Reino Unido 9 e EUA. ${ }^{8}$

Uma pesquisa no PubMed pelo termo "Teledermatology" apresenta 744 resultados, com 71 artigos publicados em 2019.

Em Portugal, a telemedicina iniciou-se legalmente com o Despacho 3571/2013 de 6 de março, identificando a dermatologia como área de implementação prioritária. Este mesmo despacho definiu erradamente o conceito de telerrastreio como sendo sinónimo de teleconsulta, conduzindo a confusão na elaboração de programas de saúde/decretos-leis subsequentes. Em abril 2014 foi publicada a Norma de
Orientação Clínica 005/2014 sob a denominação Telerrastreio Dermatológico, para melhor regulamentação. Mantendo a confusão dos conceitos de telerrastreio e teleconsulta, - Despacho 6280/2018 de 28 de junho veio tornar obrigatória a referenciação para Dermatologia com imagem e estabelece como objetivo que $80 \%$ das primeiras consultas de Dermatovenereologia sejam TD.

Atualmente, a realização de uma teleconsulta dermatológica inicia-se numa consulta nos Cuidados de Saúde Primários (CSP), com uma queixa do foro dermatológico. O médico de Medicina Geral e Familiar (MGF), através do software (Alert $\mathrm{P}^{\circledR}{ }^{\circledR}$ ) de suporte à Consulta a Tempo e Horas (CTH) faz um pedido de consulta de consulta para um Serviço de Dermatologia, anexando à informação clínica uma ou mais fotografias da lesão. O pedido é visto por um triador (dermatologista) que determina se o quadro clínico apresentado é adequado a uma teleconsulta, ou se requer uma consulta presencial. Caso opte pela primeira opção, o dermatologista responsável pela teleconsulta realiza posteriormente uma consulta não presencial, registando toda a informação (diagnóstico, eventual proposta terapêutica e recomendações gerais) na plataforma informática SClínico ${ }^{\circledR}$, no caso do Centro Hospitalar Vila Nova de Gaia/ Espinho (CHVNG/E) ou noutra, consoante o hospital. No CHVNG/E é posteriormente enviado um email à unidade de CSP, alertando o MGF responsável pelo pedido de consulta da existência da teleconsulta, para consultar o registo na plataforma SClínico-Registo de Saúde Eletrónico (RSE). Fica a cargo do MGF comunicar ao doente a informação relevante e eventualmente efetuar a prescrição sugerida pelo dermatologista (Fig. 1)

Segundo os dados da Unidade de Gestão de Acesso, ACSS, no primeiro semestre de 2018 foram realizadas 8394 teleconsultas dermatológicas, representando 18,3\% do total de consultas de Dermatologia realizadas no Serviço

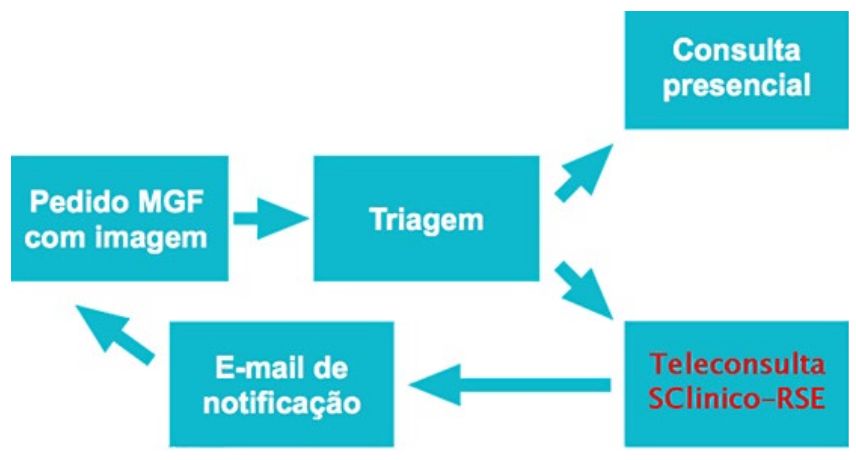

Figura 1 - Processo de referenciação hospitalar por $C T H(A l e r+P 1 \circledR)$. $\bigcirc$ processo inicia-se com o pedido do MGF. $O$ triador encaminha 0 pedido para teleconsulta. A teleconsulta é registada no SClínico ${ }$, ou outro sistema local, ficando a informação disponível para consulta pelo RSE. No CHVNG/E é de seguida enviado um email de notificação para a unidade de onde partiu o pedido, dando conhecimento da realização. Cabe depois ao MGF a comunicação da informação/terapêutica prescrita ao doente. 


\section{Artigo Original}

Nacional de Saúde (SNS). ${ }^{10}$ Assim, a imposição legal de $80 \%$ de primeiras consultas por TD revela-se de viabilidade duvidosa, e resulta da confusão entre telerrastreio e teleconsulta.

Adicionalmente, não obstante as instâncias governamentais promoverem um SNS centrado no doente, existem dados contraditórios acerca do grau de aceitação e satisfação dos doentes com esta modalidade. Embora a maioria dos estudos demonstrem aceitação e satisfação dos doentes com a TD, $1,11,12$ nenhum estudo foi efetuado na população portuguesa nesse sentido.

Assim, pretende-se com este estudo avaliar os doentes submetidos a TD no CHVNG/E durante o primeiro trimestre de 2018, bem como a perceção sobre a TD dos MGFs que trabalham em CSP na área de influência desta unidade hospitalar.

\section{MATERIAL E MÉTODOS}

Realizou-se um estudo observacional, longitudinal, retrospetivo, dividido em duas partes.

A primeira parte incluiu os doentes submetidos a uma teleconsulta de Dermatologia no primeiro trimestre de 2018 no CHVNG/E.

Todos estes doentes foram contactados por carta enviada para a morada constante no processo hospitalar a 2 de julho de 2018 (4-6 meses após a teleconsulta), solicitando- Ihes o preenchimento de um questionário (Fig. 2), composto por 10 questões e com um tempo estimado de resposta de 2 minutos. O questionário incidia sobre uma breve caracterização demográfica do doente, o conhecimento do doente acerca da existência da teleconsulta e a sua utilidade na resolução da queixa do doente, bem como a preferência do doente pela teleconsulta em função do tempo de espera para uma consulta presencial. A data limite para receção das respostas foi estabelecida a 1 de outubro de 2018. Procedeu-se seguidamente à análise retrospetiva do registo clínico dos doentes respondedores. O estudo foi aprovado pela comissão de ética do hospital.

Na segunda parte deste estudo, foi efetuado um inquérito via Google Forms ${ }^{\circledR}$ aos MGFs. A difusão do inquérito foi feita enviando um email aos coordenadores das unidades pertencentes aos Agrupamentos de Centros de Saúde (ACeS) da área de referência do CHVNG/E, pedindo a participação de todos os médicos das suas unidades. $O$ inquérito (Apêndice A) era composto por 18 questões e com um tempo estimado de resposta de 3 minutos, incidindo sobre uma caracterização demográfica do MGF, avaliação das condições materiais para a referenciação, e recolha do grau de eficácia e satisfação do MGF relativamente à TD. O prazo para receção de respostas foi entre 4 de julho e 31 de dezembro de 2018.

A recolha dos dados do estudo foi efetuada através do programa Excel. Para a análise estatística recorreu-se ao programa IBM SPSS para Windows, versão 25.0. Foi feita uma análise descritiva das variáveis em estudo. As variáveis categóricas foram descritas através de frequências absolutas (n) e relativas (\%). As variáveis contínuas foram descritas

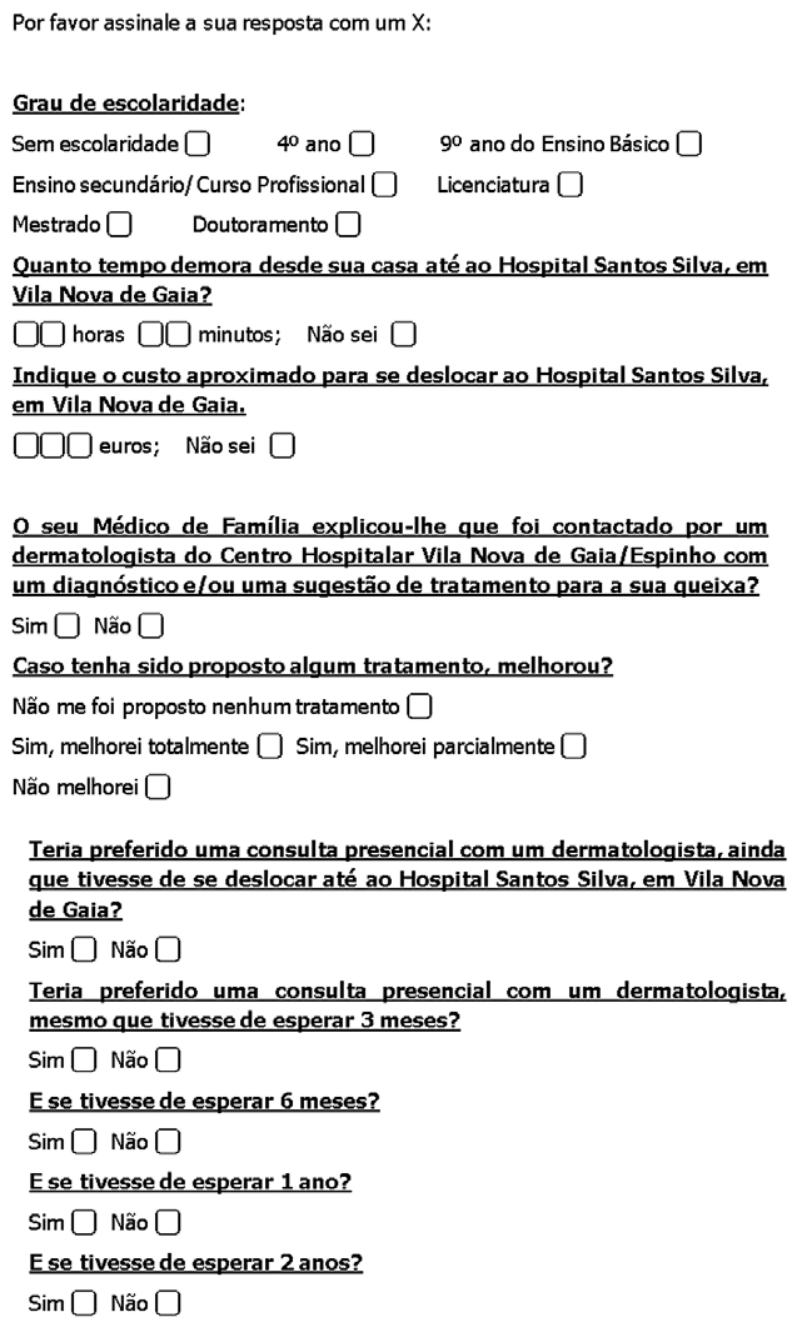

o seu Médico de Família explicou-lhe que foi contactado por um dermatologista do Centro Hospitalar Vila Nova de Gaia/Espinho com um diagnóstico e/ou uma sugestão de tratamento para a sua queixa?

Teria preferido uma consulta presencial com um dermatologista, ainda que tivesse de se deslocar até ao Hospital Santos Silva, em Vila Nova de Gaia?

Figura 2 - Inquérito enviado por correio aos doentes submetidos a TD.

utilizando a média e desvio-padrão ou mediana, percentil 25 e percentil 75 (AIQ - amplitude interquartil), consoante a distribuição destas seja simétrica ou assimétrica, respetivamente.

Foi utilizado um nível de significância de 0,05 para todos os testes de hipótese.

\section{RESULTADOS}

\section{- Inquérito realizado aos doentes}

Foram inquiridos 153 doentes, obteve-se resposta em $27,5 \%(n=42)$. Dos respondedores, $52,4 \%(n=22)$ eram do sexo masculino. A idade média foi $51,0 \pm 20,1$ anos.

Relativamente ao grau de escolaridade, $2,4 \%$ dos doentes $(n=1)$ não tinham escolaridade, $50,0 \%(n=21)$ estudaram até ao $4^{\circ}$ ano, $19,0 \%(n=8)$ tinham o $9^{\circ}$ ano, $16,7 \%$ $(n=7)$ completaram o secundário e $11,9 \%(n=5)$ apresentavam um grau de ensino superior. 


\section{Artigo Original}

\section{PATOLOGIAS DIAGNOSTICADAS}

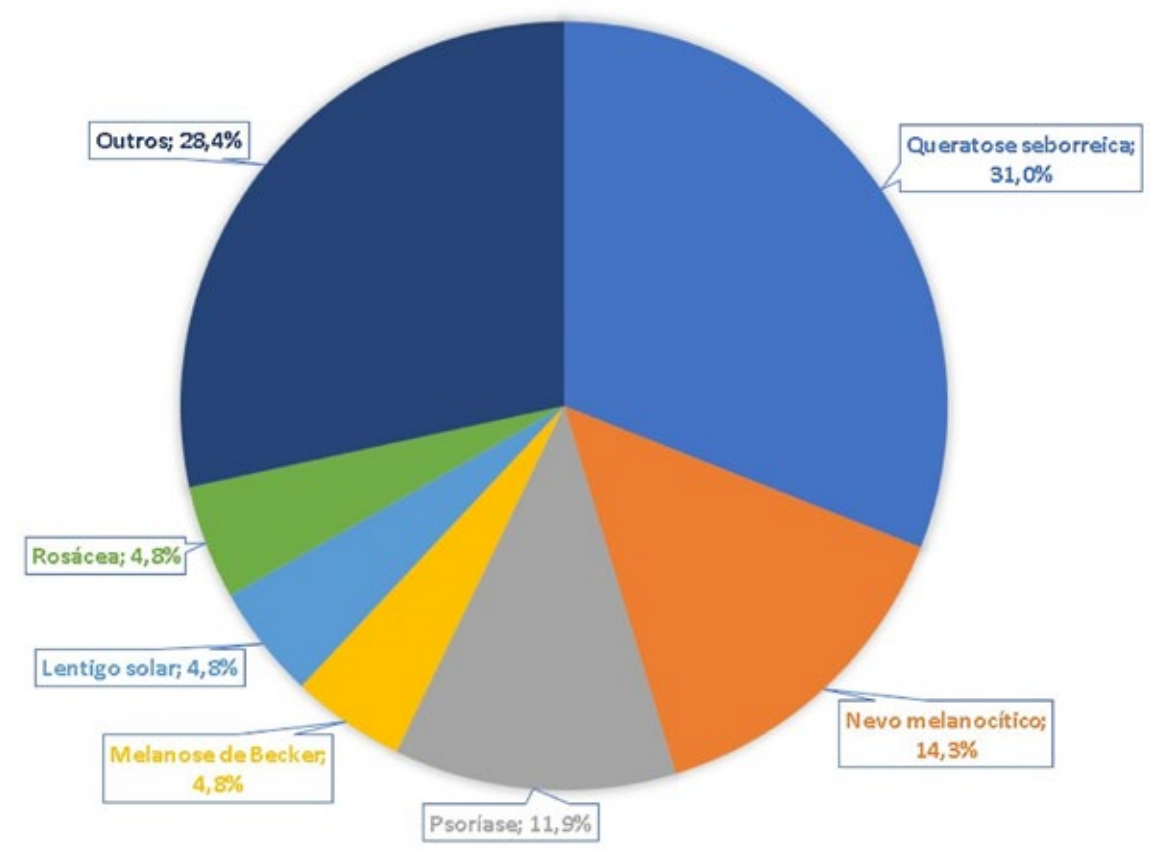

Figura 3 - Diagnósticos mais frequentes na TD

Quanto à deslocação ao CHVNG/E, a média do tempo de deslocação (ida) foi de 41,9 $\pm 27,6$ minutos, com um custo direto de $8,43 \pm 8,13 €$.

No que toca ao conhecimento do doente da TD, 73,8\% doentes $(n=31)$ afirmaram que lhes tinha sido dado conhecimento da mesma pelo MGF. Dos 11 doentes $(26,2 \%)$ que não tinham conhecimento, seis ainda não tinham sido consultados pelo MGF depois da realização da TD.

As patologias mais frequentemente diagnosticadas foram queratoses seborreicas em $31,0 \%(n=13)$, nevo(s) melanocítico(s) em 14,3\% ( $n=6)$, psoríase em $11,9 \%$ $(n=5)$, rosácea em $4,8 \%(n=2)$, melanose de Becker em $4,8 \%(n=2)$, lentigo(s) solar(es) em 4,8\% ( $n=2)$. No global, as lesões pigmentadas corresponderam a $54,8 \%(n=23)$ do total de TD (Fig. 3)

Foi proposto tratamento em $42,9 \%(n=18)$ e, neste subgrupo de doentes, $16,7 \%(n=3)$ referiram melhoria total, $44,4 \%(n=8)$ referiram melhoria parcial, $16,7 \%$ $(n=3)$ referiram não ter melhorado e $22,2 \%(n=4)$ ainda não tinham sido consultados pelo MGF depois da realização da TD, facto que pode enviesar o resultado.

Quando questionados sobre preferência por uma consulta presencial, por um dermatologista, os doentes prefeririam ser vistos em consulta presencial em 90,2\% ( $n=38)$. Analisando apenas o subgrupo de doentes com conhecimento acerca da existência da TD, 87,1\% $(n=27)$ dos doentes mantinha preferência pela consulta presencial. Contudo, quando questionados se preferiam aguardar
$3,6,12$ e 24 meses por uma consulta presencial no CHVNG/E, a taxa de respostas positivas foi de $81,0 \%$ $(n=34), 45,2 \%(n=19), 35,7 \%(n=15)$ e $33,3 \%(n=14)$ respetivamente.

Não existe diferença estatisticamente significativa no tempo de espera que os doentes estariam dispostos a aguardar por uma consulta presencial entre doentes consultados por lesões pigmentadas e doentes com outras patologias (teste do qui-quadrado, $p=0,423$ ), nem entre os doentes a quem foi sugerido um tratamento e os que não necessitaram de tratamento (teste do qui-quadrado, $p=0,254$ ) nem entre os doentes que melhoraram (parcial ou totalmente) e os doentes a quem foi instituído tratamento, mas não melhoraram (teste exato de Fisher, $p=0,083$ )

\section{- Inquérito realizado aos MGF}

Entre os vários médicos dos centros de saúde a quem foi dirigido o inquérito, e que no máximo poderia incluir cerca de 350 médicos obtiveram-se 82 respostas, das quais $73,2 \%(n=60)$ do sexo feminino, com uma idade média de $46,1 \pm 12,8$ anos.

Relativamente ao grau na carreira médica, 7,3\% $(n=6)$ eram assistentes graduados sénior, $45,1 \%(n=37)$ eram assistentes graduados, $34,2 \%(n=28)$ assistentes e $13,4 \%$ $(n=11)$ Internos de Formação Específica em Medicina Geral e Familiar.

Os médicos trabalhavam em Unidade de Saúde Familiar (USF) modelo B em 75,6\% ( $n=62)$ dos casos, em USF 
modelo A em 12,2\% ( $n=10)$ e em Unidade de Cuidados de Saúde Personalizados em 12,2\% ( $n=10)$.

Não existe câmara fotográfica em $14,6 \%(n=12)$ das Unidades onde trabalham os médicos que responderam ao inquérito, sendo que $85,4 \%(n=70)$ referem ter máquina na unidade, mas não no consultório.

Quando precisam de tirar fotografias, $69,4 \%(n=57)$ dos médicos recorrem a um telemóvel, sendo que apenas $23,2 \%$ $(n=19)$ dos médicos utilizam a câmara fotográfica da Unidade (Fig. 4)

Em média, 72,0\% ( $n=59)$ dos médicos fazem um pedido de consulta para Dermatologia por semana.

Antes da obrigatoriedade definida pelo Despacho $6280 / 2018,24,4 \%(n=20)$ dos médicos referiam que anexavam sempre imagem ao pedido, e 50,0\% $(n=41)$ dos médicos anexavam imagens em $90 \%$ ou mais dos pedidos.

Segundo estes MGFs, a referenciação com imagem acrescenta 11,2 \pm 8,8 minutos à elaboração do pedido de consulta hospitalar.

A informação de retorno (e-mail enviado para a Unidade de origem do pedido a informar que foi realizada uma teleconsulta e cujo registo pode ser consultado pelo SClínico ${ }^{\circledR}$-RSE) chega aos MGF em apenas $52,4 \%(n=43)$ das vezes. Quando a recebem, esta é satisfatória para 0 MGF em $34,9 \%(n=15)$, sendo o grau de satisfação $\geq 4$, numa escala de 1 a 5.

Curiosamente, $50 \%(n=41)$ dos MGFs concordam com a obrigatoriedade expressa no Despacho 6280/2018, mas apenas $8,5 \%(n=7)$ dos médicos consideram que a teleconsulta pode resolver pelo menos $80 \%$ dos pedidos, sem necessidade de consulta hospitalar presencial.

\section{DISCUSSÃO}

Durante $\circ 1^{\circ}$ semestre de 2018 foram realizadas no CHVNG/E 339 consultas de TD (54,2 consultas/mês, com um tempo médio de resposta de 34,1 dias), ${ }^{10}$ o que constituiu $18,3 \%$ do total de primeiras consultas da especialidade no Serviço, valor semelhante à média nacional considerada pela ACCS. ${ }^{10}$ Ainda que este estudo não seja representativo da população nacional, são possíveis algumas inferências.

Embora a maioria dos estudos revele satisfação dos doentes com a TD, ${ }^{12}$ a grande maioria dos doentes $(90,2 \%)$ da nossa área de referenciação tem preferência por uma consulta presencial. No entanto, face a tempos de espera elevados, existe uma mudança com redução da percentagem de preferências do doente pela consulta presencial se tiver de esperar 3 meses $(81,0 \%), 6$ meses $(45,2 \%), 1$ ano $(35,7 \%)$ ou 2 anos $(33,3 \%)$. A distância relativamente curta entre o domicílio do doente e o local da consulta hospitalar, com um tempo de deslocação e valor de deslocação relativamente baixos, pode influenciar pouco a decisão de preferência pela consulta presencial, de forma distinta de outras áreas do país em que a distância pode ultrapassar a centena de quilómetros e os custos das deslocações se

\section{DISPOSITIVO UTILIZADO PARA TIRAR FOTOGRAFIAS}

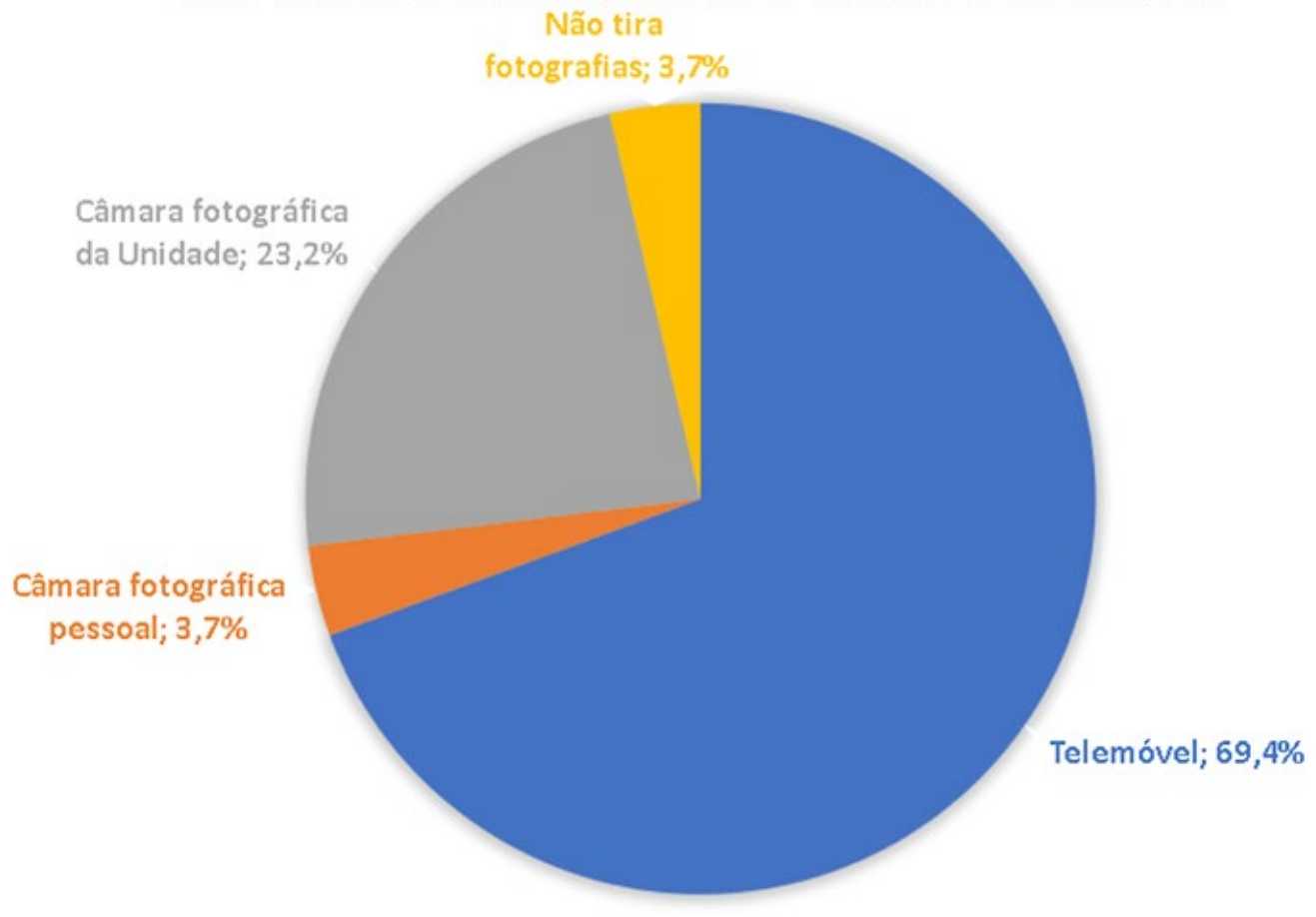

Figura 4 - Dispositivo utilizado para tirar fotografias. 
tornam elevados. A maioria dos estudos sobre esta temática avalia o grau de satisfação dos doentes per se, sem ter em conta a preferência por uma consulta presencial, nem tendo em conta os tempos de espera para a consulta (trade off entre tempo de espera e preferência de tipo de consulta). ${ }^{13,14}$ Adicionalmente, a falta de comunicação/conhecimento do doente pode afetar as opiniões dos doentes. Enquanto neste estudo apenas $73,3 \%$ dos doentes foram informados (a posteriori) que tinham sido avaliados por um dermatologista à distância, noutros estudos todos os doentes sabiam antecipadamente que iam ser vistos por teledermatologia. ${ }^{3,5,13}$ Por outro lado, não se sabe o tempo que demora desde que a teleconsulta é realizada até o doente ser informado acerca da mesma, facto que pode influenciar negativamente a satisfação do doente. Um estudo concluiu que os aspetos que mais se correlacionavam com a satisfação dos doentes com a TD eram a explicação do diagnóstico, bem como informação adequada sobre a patologia. ${ }^{13,14}$ Embora nas TD realizadas no CHVNG/E esteja sempre descrito o diagnóstico, eventual tratamento e prognóstico, apenas $34,9 \%$ dos MGFs consideram que essa informação é satisfatória.

Os resultados dos inquéritos feitos aos MGFs revelam também alguns pontos fracos da TD em Portugal, nas condições atuais. Neste estudo, quando há necessidade de tirar uma fotografia ao doente, 69,5\% dos médicos recorre a um telemóvel (provavelmente o próprio), sendo que apenas 23,2\% dos médicos utilizam a câmara fotográfica da Unidade. O uso do telemóvel para fotografar as lesões dermatológicas dos doentes, além de não ser a ferramenta ideal na captura de imagens macro, levanta problemas éticos e legais de proteção de dados pessoais, com o armazenamento em dispositivos pessoais e em nuvem, Pelo contrário, na maioria dos estudos descritos os médicos que referenciam os doentes para uma consulta de TD têm à sua disposição câmaras fotográficas dedicadas para essa tarefa, ${ }^{15}$ dermatoscópios ${ }^{5,16}$ e formação específica em fotografia dermatológica, ${ }^{17}$ fatores passíveis de melhorar a qualidade do serviço e consequentemente, o grau de satisfação dos doentes. Além disso, os MGFs referem que a referenciação com imagem (tirar foto, transferir para o computador e anexar ao pedido de consulta hospitalar) acrescenta 11,2 \pm 8,8 minutos ao tempo consumido para ultimar a referenciação, o que, a par do facto de apenas $52,4 \%$ dos MGFs receberem a informação de retorno, revela pouca otimização do processo.

Não obstante a falta de condições que os médicos de MGFs enfrentam, metade dos inquiridos concordam com a obrigatoriedade expressa no Despacho 6280/2018, o que demonstra uma consciencialização por parte dos MGFs da necessidade de fotografias para o bom funcionamento do telerrastreio e eventual teleconsulta dermatológica.

O Decreto-Lei estipula como objetivo que $80 \%$ das primeiras consultas da especialidade de Dermatologia sejam feitas por TD. Como já foi referido anteriormente, este valor deriva da confusão de conceitos entre telerrastreio e teleconsulta. Ainda que assim não fosse, a utilização da teledermatologia nunca poderá substituir a consulta presencial.
A título de exemplo, apenas $8,5 \%$ dos médicos consideram que a teleconsulta pode resolver pelo menos $80 \%$ dos pedidos, sem necessidade de consulta hospitalar presencial.

O erro inerente a esta técnica também não deve ser menosprezado. Uma revisão de um serviço de teledermatologia no Reino Unido demonstrou que em casos duvidosos existe discordância de $54 \%$ dos diagnósticos dados por teleconsulta e o diagnóstico dado em consulta presencial, e discordância de $32 \%$ entre o diagnóstico dado por teleconsulta e o diagnóstico confirmado por biópsia. ${ }^{9}$ A utilização de TD em tempo diferido permite taxas de concordância de diagnóstico de 64\% - 85\%. ${ }^{1,9}$ Embora este estudo não permita avaliar a acuidade diagnóstica da consulta de TD, 20\% dos doentes englobados neste estudo referiram não ter meIhorado nada com o tratamento, e $40 \%$ referiram ter meIhorado apenas parcialmente. Tal pode dever-se a diversos fatores tal como o carácter crónico e/ou recidivante de certas doenças, a falta de compliance dos doentes, a falta de explicação de certas medidas gerais, fatores evitáveis caso pudesse fazer-se uma consulta presencial. A existência de um grupo de controlo de doentes observados em consulta presencial permitiria comparar a eficácia terapêutica entre consulta presencial e TD.

Este estudo apresenta certas limitações. Para além da inexistência de um grupo de controlo de doentes, a possibilidade de extravio das cartas enviadas aos doentes e o número reduzido de respostas dos doentes (embora dentro do que é habitual neste tipo de inquéritos ${ }^{9,18}$ ) não permite descartar um viés de respondedor. $\mathrm{O}$ caráter retrospetivo do trabalho pode influenciar os resultados, com doentes a confundirem-se quanto à informação que lhes foi prestada em consulta com o MGF. Pelo contrário, a resposta de 82 MGFs é representativa da população, permitindo uma boa estimativa da perceção dos médicos que trabalham nos CSP acerca da TD.

\section{CONCLUSÃO}

Com o avanço tecnológico a nível de captação de imagem e de partilha de informações, a teledermatologia tornou-se uma ferramenta ao dispor do Serviço Nacional de Saúde. Face às longas listas de espera para consulta de Dermatologia, a utilização desta ferramenta, na modalidade de teleconsulta, tornou-se atrativa para a tutela. No entanto, convém não esquecer que este método, ainda que não tenha sido comparado com um grupo de controlo de doentes em consulta hospitalar, está longe de ser o ideal, quer pela preferência dos doentes numa consulta presencial $(90,2 \%)$, quer pela resolução subótima das queixas (apenas 20\% referiram melhoria total com o tratamento instituído), quer pela inerente falibilidade desta modalidade face à consulta presencial e eventuais meios complementares de diagnósticos disponíveis.

Assim, a consulta não presencial de Teledermatologia apenas permite resolver uma pequena percentagem de situações simples com escassa diminuição da lista de espera para consulta hospitalar, com menor impacto nos tempos de espera para consulta presencial, não devendo nunca ser uma substituição maciça da consulta presencial. 
Conflitos de interesse: Os autores declaram a inexistência de conflitos de interesse na realização do presente trabalho.

Fontes de financiamento: Não existiram fontes externas de financiamento para a realização deste artigo.

Confidencialidade dos dados: Os autores declaram ter seguido os protocolos da sua instituição acerca da publicação dos dados de doentes.

Protecção de pessoas e animais: Os autores declaram que os procedimentos seguidos estavam de acordo com os regulamentos estabelecidos pelos responsáveis da Comissão de Investigação Clínica e Ética e de acordo com a Declaração de Helsínquia da Associação Médica Mundial.

Proveniência e revisão por pares: Não comissionado; revisão externa por pares.

Conflicts of interest: The authors have no conflicts of interest to declare.

Financing support: This work has not received any contribution, grant or scholarship.

Confidentiality of data: The authors declare that they have followed the protocols of their work center on the publication of data from patients.

Protection of human and animal subjects: The authors declare that the procedures followed were in accordance with the regulations of the relevant clinical research ethics committee and with those of the Code of Ethics of the World Medical Association (Declaration of Helsinki).

Provenance and peer review: Not commissioned; externally peer reviewed

\section{ORCID}

Diogo Teixeira

https://orcid.org/0000-0002-5222-8192

\section{REFERÊNCIAS}

1. Coates SJ, Kvedar J, Granstein RD. Teledermatology: from historical perspective to emerging techniques of the modern era: part I: History, rationale, and current practice. J Am Acad Dermatol. 2015;72:563-74; quiz 75-6.

2. Eedy D. Dermatology: a specialty in crisis. Clin Med. 2015; 15:509-10.

3. Livingstone J, Solomon J. An assessment of the cost-effectiveness, safety of referral and patient satisfaction of a general practice teledermatology service. London J Prim Care . 2015;7:31-5. doi: 10.1080/17571472.2015.11493433.

4. Kimball AB, Resneck JS, Jr. The US dermatology workforce: a specialty remains in shortage. J Am Acad Dermatol. 2008;59:741-5. doi: 10.1016/i.jaad.2008.06.037.

5. Hue L, Makhloufi S, Sall N'Diaye P, Blanchet-Bardon C, Sulimovic L, Pomykala F, et al. Real-time mobile teledermoscopy for skin cancer screening targeting an agricultural population: an experiment on 289 patients in France. J Eur Acad Dermatol Venereol. 2016;30:20-4. doi: $10.1111 /$ idv. 13404 .

6. Tandjung $R$, Badertscher $N$, Kleiner $N$, Wensing $M$,
Rosemann T, Braun RP, et al. Feasibility and diagnostic accuracy of teledermatology in Swiss primary care: process analysis of a randomized controlled trial. J Eval Clin Pract. 2015;21:326-31. doi: 10.1111/jep.12323.

7. Romero G, de Argila D, Ferrandiz L, Sánchez MP, Vañó $\mathrm{S}$, Taberner $\mathrm{R}$, et al. Practice Models in Teledermatology in Spain: Longitudinal Study, 2009-2014. Actas Dermosifiliogr. 2018;109:624-30. English, Spanish. doi: 10.1016/j.ad.2018.03.015.

8. Tensen E, van der Heijden JP, Jaspers MW, Witkamp L. Two Decades of Teledermatology: Current Status and Integration in National Healthcare Systems. Curr Dermatol Rep. 2016;5:96-104. doi: 10.1007/s13671016-0136-7.

9. Mehrtens SH, Shall L, Halpern SM. A 14-year review of a UK teledermatology service: experience of over 40000 teleconsultations. Clin Exp Dermatol. 2019;44:874-81. doi: $10.1111 /$ ced.13928.

10. ACSS.Unidade de Gestão de Acesso, ACSS, $1^{\circ}$ semestre 2018. Lisboa: ACSS; 2018.

11. Whited JD. Teledermatology. Med Clin North Am. 2015;99:1365-79, xiv. doi: 10.1016/j. mcna.2015.07.005.

12. Mounessa JS, Chapman S, Braunberger T, Qin R, Lipoff $J B$, Dellavalle RP, et al. A systematic review of satisfaction with teledermatology. J Telemed Telecare. 2018;24:26370. doi: 10.1177/1357633X17696587.

13. Hsueh MT, Eastman K, McFarland LV, Raugi GJ, Reiber GE. Teledermatology patient satisfaction in the Pacific Northwest. Telemed J E Health. 2012;18:377-81. doi: 10.1089/tmi.2011.0181.

14. Qureshi AA, Brandling-Bennett HA, Wittenberg E, Chen SC, Sober AJ, Kvedar JC. Willingness-to-pay stated preferences for telemedicine versus in-person visits in patients with a history of psoriasis or melanoma. Telemed J E Health. 2006;12:639-43. doi: 10.1089/ tmi.2006.12.639.

15. Marchell R, Locatis C, Burgess G, Maisiak R, Liu WL, Ackerman M. Patient and Provider Satisfaction with Teledermatology. Telemed J E Health. 2017;23:684-90. doi: 10.1089/tmi.2016.0192.

16. Barcaui CB, Lima PM. Application of Teledermoscopy in the Diagnosis of Pigmented Lesions. Int J Telemed Appl . 2018;2018:1624073. doi: 10.1155/2018/1624073.

17. van der Heijden JP, de Keizer NF, Bos JD, Spuls PI, Witkamp L. Teledermatology applied following patient selection by general practitioners in daily practice improves efficiency and quality of care at lower cost. $\mathrm{Br}$ J Dermatol. 2011 ; 165:1058-65. doi: 10.1111/j.1365$-2133.2011 .10509 . x$.

18. Rajda J, Seraly MP, Fernandes J, Niejadlik K, Wei H, Fox K, et al. Impact of Direct to Consumer Store-and-Forward Teledermatology on Access to Care, Satisfaction, Utilization, and Costs in a Commercial Health Plan Population. Telemed J E Health. 2018;24:166-9. doi: 10.1089/tmi.2017.0078. 